\title{
16. COMMISSION POUR LES OBSERVATIONS PHYSIQUES DES PLANETES ET DES SATELLITES
}

Président : M. G. P. Kuiper.

Membres: MM. Adel, Alexander, Baldwin, Barabashev, Camichel, Danjon, Delporte $\dagger$, Dollfus, de Vaucouleurs, Dunham, Fournier $\uparrow$, Gentili, Gialanella $\uparrow$, Giclas, Harris, Heath, Herzberg, Letfus, Lipsky, Luplau-Janssen, Nicholson, Peek, Pettit, Plakidis, Ryves $\dagger$, Schoenberg, Sharonov, E. C. Slipher, Steavenson, Mme Sytinskaya, MM. Tikhov, Urey, Weimer, Wildt, Wilkins, F. E. Wright $\dagger$.

\section{i6a. Sous-commission de la Nomenclature Martienne}

Président: M. G. Fournier $\dagger$.

Membres: MM. Alexander, Barabashev, Camichel, Kuiper, van Biesbroeck.

We report with deep regret the deaths of Georges Fournier, L. Gialanella and Frederick E. Wright. M. Fournier, together with Jarry Desloges, published the monumental series of Io volumes Observations des surfaces planétaires, begun in 1907. During the months before his death he worked on a revision and simplification of Martian nomenclature, in furtherance of a decision made by this commission at the Rome meetings. The status of this work is reviewed in the Appendix to this Report.

\section{NAME OF THE COMMISSION}

\section{Recommendation by G. P. Kuiper:}

It is proposed that the name of the Commission be changed by replacing 'Observations' by 'Etudes'; this change would recognize theoretical studies of planetary structure and associated laboratory work as part of planetary research.

Replies received to a circular letter addressed to the members indicate nearly unanimous agreement with this proposal.

\section{PROGRESS OF RESEARCH}

\section{Diameters}

The period covered by this report has brought to a near-conclusion several series of high-precision diameter measurements, mostly carried out in France. The invention of the disk meter by Lyot and Camichel and its use on the $60 \mathrm{~cm}$. refractor at the Pic du Midi have led to an important series of measures by Camichel (r). Muller, with a doubleimage micrometer on the $16 \mathrm{~cm}$. refractor at Strasbourg has measured Mercury, Venus, Mars, Uranus and Titan (all but two measurements made prior to I952 and mostly published). Dollfus (2), with Lyot's double-image micrometer on the $60 \mathrm{~cm}$. refractor, has measured the large satellites of Jupiter, Titan and Neptune. With a new doubleimage device of large separation he has measured Mars (3) in yellow light $\left(9^{\prime \prime} \cdot 25 \pm 0\right.$ "03 at I astr. unit) and blue light $(9 " 40)$, as well as the oblateness in yellow light (0.01 $3 \pm 0.006)$. Of special interest is his measure of Mercury (4) when it was projected on the Sun on I4 November I953, made from the top of the Eiffel Tower in Paris. He used a $16 \mathrm{~cm}$. refractor and a double-image micrometer; the result, obtained under somewhat difficult conditions, was $6 " 45$ at I astr. unit, compared to Muller's value of $6 " 42$ (I5 days, I939-5I). The average value makes the density of Mercury about $6 \cdot I$.

Kuiper (5) used a disk meter on the 82 in. telescope to measure four Jupiter and four Saturn satellites and Neptune; further, with the 200 in. telescope, Pluto (6) and (on 5 July I954, unpublished) Enceladus (0"08), Tethys ( $\left.0^{\prime \prime} \mathrm{I} 2\right)$, Dione (0"I2), Rhea (0"24), Titan (0"67), Iapetus ( 0.195$)$, all at 9.43 astr. units; and Triton ( 0 "I73) and Neptune $(2 " 06)$, both at $30 \cdot I$ astr. units. The suggestion has been made ( 7$)$ that Pluto might show a spurious disk owing to specular reflection. That this is most unlikely was shown by 
Kuiper (8); furthermore, with Pluto 5 mag. above the threshold of the telescope, a strong limb darkening would have been seen. The disk of Pluto is now known to have been at least five times the effective resolving power used. Finally, evidence has been found ( 9 ) for an atmosphere on Pluto which in itself will prevent the edge of the planet from being dark. The small size of Pluto as measured is therefore regarded as reliable within the stated limits of precision. This makes the case of Triton of special interest, as being similar though somewhat smaller than Pluto. The density of Triton now found, $3 \cdot 2$, is at present uncertain primarily because of the mass $( \pm 18 \%)$; a new determination of it will therefore be important. Kuiper also measured Uranus and Mars (double-image micrometer); and Venus, using the cusps at inferior conjunction. Further, the diameter difference, blue-red, for Mars; this difference was found to be $0.9 \%$. Nefedjev (xo) reports variations in the Venus diameter of about 0.3 , based on heliometer measures in r $94{ }^{\circ}-5 \mathrm{I}$.

\section{Rotation}

Camichel $(x)$ measured the oblateness of Mars photographically and found it to be 0.017 , confirming earlier results by Muller (0.015) and others. Since the oblateness of the solid surface must be close to the dynamical oblateness of I/I92, the measured value must refer to the top of the atmospheric haze layer, higher in the tropics than over the poles. (12)

Ashbrook (13) made a careful redetermination of the period of rotation of Mars and, incidentally, of the position of the pole. Camichel(ri) made a precise determination of the position of the Martian pole from a long series of photographs. Kuiper(14) located the pole of Venus; he found the obliquity to be about $32^{\circ}$. Dollfus ( 15 ) found the rotation of Mercury closely synchronized with its revolution, the ratio of the periods differing by less than $10^{-4}$ from unity. He found the obliquity of Mercury to be about $7^{\circ}$.

For the rotation of the earth and its variations reference is made to Commission 7 . The present status of the problem is also reviewed by Spencer Jones (x6).

\section{Photometry, colorimetry, and polarization}

Magnitudes can now be measured easily and accurately from 0.32 to $I \mu$, and with special equipment to $2 \mu$; and because the planetary albedos and colours are quite dissimilar, a great field of planetary study has opened up. So far only a few results have been published. Woolley (r7) measured Mars in 1952 between $\lambda \lambda 4050$ and 6360 . Giclas ( 18 ) determined the photo-electric magnitude and colour of Uranus. Schoenberg determined photographically the total brightness of Saturn and Ring in three colours during six oppositions, in order to study phase effects of the Ring; he anticipates these effects to be wave-length dependent. Miss Groeneveld and Kuiper (19) published colour equivalents of satellites and some planets, showing that the Jupiter satellites have no appreciable atmospheres, but that Titan, Triton and Pluto very probably do. Harris and Kuiper have, during the last few years, made a systematic photo-electric study, usually in three colours, of the planets and the satellites brighter than $m=14$, except for the Mars satellites which were observed photographically. Currently Kuiper is extending this work to $2 \mu$. The modern measures, combined with recalibrated earlier photo-electric series, give no evidence whatever for the reality of the announced long-period intensity fluctuations of several planets. The results of this survey are being prepared for publication in The Solar System, Vol. 3.

An application is the problem of the nature of the Venus clouds. Menzel and Whipple (20) favour the hypothesis that the clouds are water droplets which then, from Lyot's polarization curve, should be roughly $2 \mu$ in size. Kuiper(2x) holds that the clouds are dust, since they are yellowish and since fine haze when seen from above is white or bluish white. The absence of fine-structure in the Venus cloud cover (apart from the broad, fuzzy belts), the absence of $\mathrm{O}_{2}$ which water vapour must produce photochemically (with $\mathrm{H}$ escaping), and the great abundance of $\mathrm{CO}_{2}$ are compatible with the dust hypothesis. The dust might in part be composed of salt particles.

Sharonov (22) developed a method for comparing visually planetary colours with the 
Sun. He also published a survey of planetary and satellite photometries(23). Barabashev (24) found changes in the colour of Venus. Parshin (25) found that the reflecting layer of Venus follows the cosine law, with the following albedos: 0.67 photographic, 0.93 red, and 0.95 infra-red. Tchekirda currently measured the limb of Jupiter photometrically. Barabashev and Tchekirda (26) published photometric data on Saturn and its ring, observed in I95I.

The polarization studies of planets are all due to Dollfus and, in I954, his associate Focas of Athens, who observed Mars at the Pic du Midi. Dollfus (27) measured the polarization of the earth light on the Moon and derived the polarization curve for the Earth, a remarkable achievement. He further studied the coefficient of depolarization of the lunar surface, and deduced from laboratory comparisons that only fine powders and volcanic ash behave that way. Mars was observed in 1952 and I954; Dollfus found the morning and evening haze on Mars to correspond to droplets $2-3 \mu$ in diameter; he and Focas have accumulated data on the seasonal development of the Martian haze and clouds.

\section{Eclipses and occultations by planets}

The occultation by Jupiter of Sigma Arietis on 20 November 1952 was observed photo-electrically by Baum and Code (28) and on motion pictures by Pettit and Richardson (29). Baum and Code derive the important result that the mean molecular weight of the Jupiter atmosphere is about 3. Photo-electric photometry of eclipses of the four Jupiter satellites has been carried out by Harris and Kuiper. Photo-electric observations of a lunar eclipse have been published by Reaves and Walker (30), and measures with a 'cat-eye' photometer by Dubois (31). The shape of the earth's shadow on the moon during the eclipse of 26 . September 1950 was considered by Bouška and Ruzickova (32). On the basis of photo-electric observations, the same was done for the eclipse of 8 December I 946 by V.P. Dzhapiashvili (33). Observations of the lunar eclipses of I950-52 by Markov and Schegolev (34) were reduced. V. M. Tchernov (35) investigated data on nineteen eclipses and found the brightness of the eclipse to depend on geographic latitude and phase. Lunar occultations are being used for geodetic purposes (36). Occultations and eclipses by the Rings of Saturn have not been observed recently, and would be of great interest, particularly for the study of the Crepe Ring.

\section{Spectra and temperatures}

McKellar obtained high-dispersion spectra of Jupiter (4.2 $\AA / \mathrm{mm}$.) to test the presence of the free radical $\mathrm{NH}_{2}$ in absorption, by using the bands near $\lambda \lambda 5976$ and $6300 \AA$, observed by Herzberg and Ramsey in the laboratory (see p. 253). McKellar found no evidence of these lines in Jupiter. Hess (37) studied the strength of the absorptions $\lambda 6$ I9o of $\mathrm{CH}_{4}$ and $\lambda 644 \mathrm{I}$ of $\mathrm{NH}_{3}$ over the disks of Jupiter and Saturn; he concluded that the cloud surface on Jupiter rises toward the limbs, but is more nearly level on Saturn. Kuiper (38), using similar data, concluded that the cloud layer on Jupiter is probably level, but that its upper boundary is diffuse so that the secant effect is nearly absent in the observed absorptions. Hess found indications that the poles of Jupiter are colder than the equator and the morning side colder than the evening side. Dollfus (39) attempted to measure the water-vapour content of Mars from a free balloon at $6.5 \mathrm{~km}$. elevation; the amount was below the threshold of the method. This is consistent with the low humidity computed by Kuiper (40). Grandjean and Goody (4I) redetermine the $\mathrm{CO}_{2}$ abundance on Mars from Kuiper's (42) measures of the $I \cdot 6 \mu$ bands, by allowing theoretically for the pressure difference with the Earth. Kuiper (unpublished) has made laboratory measures for the same purpose. He has continued to build up his collection of planetary and satellite spectra for the accessible spectral range. This material and accompanying laboratory results are being prepared for publication in Vol. 3 of The Solar System. The absorption spectrum of the terrestrial atmosphere has been reviewed in detail by Goldberg (43). 
Strong and Sinton have observed Venus and Mars radiometrically with the Ioo and 200 in. reflectors. Adel has established the Atmospheric Research Observatory at Flagstaff, Arizona, studying the infra-red atmospheric spectrum and, in particular, the temperature of the ozone layer.

\section{Associated laboratory spectroscopy}

A communication received from Dr Herzberg is reproduced in full:

In 1949, Kuiper observed a diffuse band at $8270 \AA$ and a series of diffuse lines near $7500 \AA$ in the spectrum of Uranus. The first feature has been identified by laboratory experiments as due to the $\mathrm{H}_{2}$ molecule (G. Herzberg, $A p . J$. 115, 337, 1952). The observed diffuse feature represents the $S(O)$ line of the 3-o pressure-induced rotation-vibration band of the $\mathrm{H}_{2}$ molecule. A comparison between planetary and laboratory spectra indicates an amount of about $40 \mathrm{~km}$. atm. of hydrogen above the reflecting layer of Uranus with a $\mathrm{H}_{2}$ partial pressure of the order of $2 \mathrm{~atm}$. at the bottom of this atmosphere. The laboratory investigations suggest strongly that there are still larger amounts of helium in the Uranus atmosphere, probably more than three times as much. It is to be noted that only at the temperature of liquid nitrogen is the line width of the $\mathrm{H}_{2}$ line comparable to that observed in Uranus. At room temperature the width is much larger.

A number of laboratory experiments have been carried out to identify the Kuiper $7500 \AA$ band. Silane $\left(\mathrm{SiH}_{4}\right)$ and methyldeuteride $\left(\mathrm{CH}_{3} \mathrm{D}\right)$, both of which might be expected to be present in the atmospheres of the outer planets, have been studied (J. A. Bardwell and G. Herzberg, $A p . J .117,462,1953)$, but no absorption band near $7500 \AA$ was found and no evidence for any other bands of these two gases observed in the laboratory is found in planetary spectra.

In addition, the spectra of the free radicals $\mathrm{NH}_{2}$ (G. Herzberg and D. A. Ramsay, J. Chem. Phys. 20, 347, I952; Disc. Faraday Soc. I4, II, I953) and HCO (D. A. Ramsay, J. Chem. Phys. 2I, 960, 1953; G. Herzberg and D. A. Ramsay, unpublished), as well as the corresponding deuterides, have been obtained by the flash photolysis method and have been studied in considerable detail. The $\mathrm{NH}_{2}$ spectrum consists of a large number of very fine irregularly placed lines. It appears quite possible that these lines will be found in the spectra of Jupiter and Saturn once these are taken with a sufficiently high resolution, since $\mathrm{NH}_{3}$ is known to be present in the atmospheres of these planets and since photo-dissociation of $\mathrm{NH}_{3}$ must certainly occur. Thus far, however, no evidence of the $\mathrm{NH}_{2}$ lines in the planetary spectra has been found. In the case of $\mathrm{HCO}$ and $\mathrm{DCO}$, a number of very simple absorption bands has been found forming a simple progression in each case extending through the visible region. In DCO, in addition, a somewhat more complicated band consisting of a number of sub-bands has been found at $7500 \AA$. The analogue of this band in HCO has not yet been found in the laboratory. However, its structure can be predicted from that of DCO and agrees in a striking way with the observed structure of the Kuiper band at $7500 \AA$. The position of the Kuiper band is not incompatible with a reasonable vibrational isotope shift in going from DCO to $\mathrm{HCO}$. If $\mathrm{HCO}$ were really the molecule responsible for the Kuiper band, it would follow that a certain amount of $\mathrm{CO}$ must be present in the atmosphere of Uranus which reacts with the $\mathrm{H}$ atoms formed by photodissociation of methane to yield HCO. Conversely, HCO photodecomposes readily into $\mathrm{H}+\mathrm{CO}$.

The $\mathrm{CO}_{2}$ bands first observed in the spectrum of Venus have been reproduced in the laboratory a number of years ago (G. Herzberg, The Atmospheres, p. 406). These, as well as additional bands in the photographic infra-red, have now been analysed in considerable detail (G. Herzberg and L. Herzberg, J. Opt. Soc. Amer. 43, 1037, 1953).

A number of laboratory investigations carried out at Ottawa have a bearing on phenomena in the upper atmosphere of the Earth. A detailed fine-structure analysis of the forbidden ultra-violet $\mathrm{O}_{2}$ bands previously found (G. Herzberg, Naturwissenschaften, 20, 577, 1932) have now been investigated under high resolution and with a very long absorbing path (G. Herzberg, Canad. J. Phys. 30, 185, I952), and, in addition, two new forbidden absorption systems of oxygen have been found (G. Herzberg, Canad. J. Phys. 3I, 657, 1953). The former bands have recently been definitely identified in the spectrum of the night-glow (Chamberlain, $A P . J$. 
12x, 277, 1955). A study of the theoretical intensity distribution in the rotation-vibration spectrum of $\mathrm{OH}$ found by Meinel in the night-glow has been made (H. S. Heaps and G. Herzberg, $Z$. Phys. 133, 48, 1952). The new infra-red $\mathrm{N}_{2}+$ bands corresponding to the transition ${ }^{2} \Pi-2 \Sigma$ first found by Meinel in the spectrum of the aurora have been observed in the laboratory (A. E. Douglas, $A p . J .117,380,1953$ ) and the fine-structure of the bands has been analysed confirming Meinel's identification.

A summary on forbidden transitions in the spectra of diatomic molecules has been presented to the Royal Society of Canada (G. Herzberg, Trans. Roy. Soc. Can. 46, I, I952).

\section{Visual and photographic studies of surface detail}

This traditional field of planetary astronomy has seen great activity during the period of this Report, with results of special importance coming from the research centre founded by the late Dr Lyot. In an address (44) delivered I6 May I95I he gave a summary of the work with the $60 \mathrm{~cm}$. refractor at the Pic du Midi that will be as memorable as his doctoral thesis 24 years earlier. It gives an unparalleled study, including maps, of the Galilean satellites of Jupiter; photographs of Jupiter and Saturn; and drawings of Saturn, its Rings, and Neptune. This remarkable address is supplemented by two important papers by Dollfus (45), describing the results obtained on Mercury and Venus, and Mars, respectively. Mercury was studied photographically in I942 and I944, leading to the construction of a valuable photographic map (46). Visual studies by Lyot in I942, and particularly by Dollfus in I950, have added greatly to our knowledge of the surface markings. The existence of veiles, so often reported before, was not confirmed. Dollfus' studies also led to important data on the rotation of Mercury and its obliquity (see p. 25I) and on its atmosphere. The study of Venus was based on a hundred plates taken in yellow light, only some of which showed distinct markings; a few composite photographs with increased contrast are reproduced.

Dollfus' study of Mars extended over the years 1945 to 1952. Noteworthy features are the secular changes portrayed, the effect of seeing on the appearance of the Syrtis Major region, and the disappearance of various types of canals as the seeing improves. From the contours of the waning North polar cap he derived elevation differences between the snow-covered plateaux and their surroundings of about $\mathrm{I} \mathrm{km}$. The dark areas have the property of being darkest just after they have lost their snow cover. Dollfus further comments on the types and frequency of clouds and haze. Drawings and notes on the I952 opposition of Mars, made by several observers with 3 and ro in. telescopes, were published by Fournier (47). Kuiper measured visually the colours of Martian dark areas with the 82 in. telescope during June-July r954, using a binocular attachment with power $900 \times$. The apparent colour was found to depend on seeing; for example, Mare Acidalium appeared greenish with mediocre seeing, but lost this colour as the seeing improved. With perfect seeing all maria appeared merely darker than the 'deserts', but essentially of the same colour, throughout the entire period. Kuiper confirms Dollfus's statement on the absence of long, narrow canals. This subject is unique in astronomy, in that a result obtained half a century ago by Barnard, Antoniadi, Hale and others, seems to require periodic reaffirmation.

Two developments of importance are recorded. Humason has taken a few experimental photographs of planets with the $200 \mathrm{in}$., at the $500 \mathrm{ft}$. coude focus, that show the potential power of this great instrument also in this field. Strong and Sturm have applied television techniques to the photography of planets. They used an image orthicon on the 24 in. refractor of the Lowell Observatory on Mars in I954, and obtained with an experimental arrangement oscilloscope photographs that are already somewhat superior to direct photographs with the same telescope, apparently because of the increased speed.

Tikhov reports that an associate, M. Koutcherov, observed Mars in 1954 from Abastumani Observatory with a $\mathrm{r} 6$ in. refractor. He obtained 70 drawings using filters, and about 700 photographs, also with filters. Tikhov sees in the results a confirmation of his general ideas ${\left(4^{8}\right)}^{8}$ published early in I953. Other associates examined the optical properties 
of plants. Dr Tikhov remarks that this work may have received insufficient attention outside the U.S.S.R.; he summarized it in two small books, Astrobotany and Astrobiology, published in $\mathrm{I} 949$ and $\mathrm{r} 953$, respectively.

Sharonov (49) discussed the visibility of planetary detail. Barabashev and Tchekirda (50) studied Mars in I952 and found the polar cap reddish; Barabashev (5x) found most of the maria reddish also. Bobrov(52) found Ring B of Saturn to have a small volume density (about $\mathrm{IO}^{-3}$ ), with particles of high albedo. M. V. Bannova (53) published isophotes of Saturn.

E. C. Slipher, of the Lowell Observatory, took an extensive series of Mars photographs in I954 during an expedition to Bloemfontein, South Africa. Leighton (54) reported on the advantages of using colour film in planetary photography with the $60 \mathrm{in}$. Mt Wilson reflector. Martz (55) reproduced some I939 photographs of Mars and discussed the haze layer. Dollfus (56) examined the relative merits of visual work and photography of planets and the best evaluation procedure for photographs. McLaughlin (57) advanced the hypothesis that the Martian surface markings are due to volcanic deposits, distributed by zonal winds. Some comments on this hypothesis are made by Kuiper (58). Schoenberg (59) studied the equatorial acceleration of Jupiter. He further collected all measurements of the jovicentric latitudes of five dark belts (60). Luplau-Janssen continued his micrometric latitude measures of the belts, begun in I9I4. Observations of Jupiter by members of the B.A.A. are currently being published in the Journal of the B.A.A., instead of the Memoirs, as were oppositions prior to I943.

Kuiper, on a nearly perfect night in I954, examined the Rings of Saturn and the planet Neptune with the 200 in. telescope, with power II70 $\times$. He had been doubtful that spots (i.e. clouds) could be visible on Neptune, as reported, because of the atmospheric model required to yield the observed strengths of methane and hydrogen (6r). Actually no spots could be seen on a beautifully defined disk that showed appreciable limb darkening (estimated intensity of limb, 0.6 of centre).

The rings of Saturn were examined for the reality of the numerous 'divisions' that had been reported. Only one division exists, the Cassini division, whose width is one-fifth of that of Ring A. The other 'divisions' are either minor intensity ripples, with some IO-I $5 \%$ amplitude, or are non-existent. The Encke 'division' is a ripple where at the same time Ring A changes its intensity abruptly. There are three ripples in Ring B, and there is no gap between Ring B and the Crepe Ring. There is an incredible difference between the large-scale luminous image of the great telescope, without disturbing chromatic effects, and with an effective resolving power of $0^{\prime \prime} \mathrm{O} 5$ or better, and that seen in a 20 or 40 in. refractor. The intensity profile of the Ring, including the Crepe Ring, was measured with a double-image device on the 82 in. telescope.

Dollfus and de Mottoni are currently evaluating the 4I5 Mars plates, taken at Pic du Midi by Camichel since I94I, for seasonal and secular changes. Kuiper (62) is studying the latitudes of the six to eight Venus belts and the effects of the large obliquity.

\section{Lunar studies}

The upper limit of the lunar atmosphere established by Dollfus $\left(6_{3}\right)$ is $10^{-0}$ of the terrestrial atmosphere, so that the density at the lunar surface is less than that in the terrestrial atmosphere at $\mathrm{I} 8 \mathrm{o} \mathrm{km}$. elevation, far above the meteor zone. One is therefore inclined to attribute reports on lunar meteors to terrestrial telescopic meteors instead. On very rare occasions explosions on the moon might perhaps be visible (64). Lunar eclipse observations are referred to on p. 252.

A study of the lunar surface features with the 82 in. telescope and of the origin of these features was published by Kuiper (65). Buettner (66) considered erosion of the lunar surface. Wesselink (67) reviewed the present status of the knowledge of the lunar surface material derived from thermal and radio data. The thickness of the dust layer on the moon is not yet known; only radio data for small regions of the lunar surface can give new information. 
Photometry and colorimetry of lunar surface detail has again been actively pursued in the U.S.S.R. V. A. Fedoretz (68) completed her differential photometry of 172 small areas, over phase angles $I^{\circ} \cdot 5$ to $I_{5} 0^{\circ}$. N. N. Sytinskaya (69) summarized all published visual measures and published a catalogue of absolute albedos for ro4 lunar details. N. P. Barabashev and A. T. Tchekirda (70) determined colours of lunar formations with the aid of filters. A. V. Markov (7x) reported changes in the reflectivity of certain areas, e.g. crater Plato. N. N. Sytinskaya (72) compared visual estimates of brightness, on Pickering's scale, with absolute albedos. V. V. Sharonov (73) studied the effects of temperature changes caused by shadows. N. S. Orlova (74) investigated the diffuse reflection of light by terrestrial minerals. A. P. Borissova and N. A. Budnikova (75) measured the albedo and colour of a large sample of rocks and meteorites. V. V. Sharonov (76) compared the spectral reflection curves of different terrestrial rocks and meteorites with the Moon, and found no similarity. V. P. Dzhapiashvili made photo-electric polarization measures of over forty lunar formations, with the maria giving the highest values. Similar work was done at Pulkovo Observatory by A. V. Markov (77). J. N. Lipsky developed new spectrophotometric equipment also suited for the measurement of polarization as a function of wave-length. V. I. Müchkulia constructed a new type of self-recording polarimeter for lunar details.

\section{Asteroids}

The high-precision photometry of individual asteroids initiated at the McDonald Observatory in I 949 has so far led to four articles on 'Photometric Studies of Asteroids', by Groeneveld and Kuiper (I and II) (78), Shatzel (III) (79), and Ahmad (IV) (80). It was found that light variations occur in at least $90 \%$ of the asteroids, and that those of appreciable amplitude are due to changes in projected area rather than spots; that the periods of rotation at least range from 4 to $20 \mathrm{hr}$.; and, very provisionally, that the axes of rotation are oriented roughly at random. Eunomia, no. 15, was the first body found to have a definite retrograde rotation (i.e. one with low obliquity, unlike Uranus).

The chief objective of the McDonald Observatory Asteroid Survey (8r) to $m=16 \cdot 5$, also begun in I949, was to determine photometric magnitudes of all asteroids down to $m=I 6$ and to determine the absolute number of asteroids in each magnitude interval covered. With the reductions more than half complete the provisional statement may be made that between $m=10$ and $I 6$ the number of asteroids at opposition (on a photometric scale and freed from selection effects) increases by a factor of very nearly 2 for each fainter magnitude. This means that the total mass contribution of each fainter magnitude decreases by about 2 . The interval $m=I 6-19$ is also under study, from different plates; the preliminary impression, that the numbers increase somewhat faster in this interval, needs confirmation.

B. J. Levin (82) examined the constants in the equation for determining asteroid dimensions photometrically. The problem of the origin of the asteroids was considered by Kuiper (83) and O. J. Schmidt (84). E. A. Lubimova and A. S. Starkova (85) examined the heating of asteroids and showed that for bodies of $200 \mathrm{~km}$. in diameter and larger, the past temperature could have exceeded $1000^{\circ}$. Similar results were obtained by Kuiper (86). The important related subject of the physics and chemistry of meteorites is not at present covered by Commission Reports, but deserves to be in the future.

\section{Physics of planetary atmospheres}

A monograph by N. P. Barabashev (87) summarizes his studies in this field. S. D. Gutshabash (88) deduced an approximate and a precise solution of the penetration and scattering of light in a level layer of a turbid medium. V. V. Sobolev (89) published an article on 'Theory of Transfer of Light in Planetary Atmospheres', containing tables for the calculations. V. V. Sharonov (9o) found that the horizontal refraction on Venus to be $2 \mathrm{O}^{\prime \prime}$, and interpreted this low value by assuming the presence of a thin translucent cloud layer (gr). Reference should also be made to The Atmospheres of the Earth and Planets, to The Earth as a Planet, chs. 7-15 and to Urey's The Planets. 


\section{Planetary interiors}

The accurate mass determination for Mercury by E. Rabe (92) made it clear that the widespread assumption of equality of composition among the terrestrial planets was probably invalid; the only reservation was that the diameter of Mercury was still somewhat uncertain (93). The improved diameter of Mercury (see p. 250) makes the argument conclusive. The inequality of composition of the terrestrial planets, with Mercury possessing as much as three-quarters metal phase by weight, must be regarded as a cornerstone in future studies of composition and origin of the terrestrial planets.

The internal structure of the Earth has been reviewed by Jeffreys (94), Bullen (95), Bullard and Mason (96), and others. The structure of the Moon is discussed by Jeffreys (op. cit.); its structure and thermal history by Kuiper (97). The structure of the Jovian planets was analysed by Ramsey (98); and of Jupiter and Saturn, specifically, by Miles and Ramsey (99), and De Marcus and Wildt (unpublished). De Marcus (roo) studied the outer layers of Jupiter and Saturn. Bernal and Massey (xor) derived the pressure-density relation for metallic ammonium; Ramsey had pointed out the likelihood that this substance is an important constituent of Uranus and Neptune. The new calculations lend weight to this conclusion. Some comments on the composition of the satellites, inferred from their densities, were made by Berlage (ro2), Urey (ro3) and Kuiper(xo4). De Marcus (ro5) published a study 'On the instability of small cores in rotating planets'.

S. V. Kozlovskaya (ro6) drew attention to the analogy between the terrestrial planets and the large Jupiter satellites, with the densest members inside. E. A. Lubimova derived analytically the heating of the Earth by radioactivity, on a uniform model (ro7); and a layered model with crust, mantle and core (ro8). Radioactive decay was taken into account. Jacobs (rog) has also studied the problem of the cooling of the earth.

\section{Problems of origin}

In the last few years many divergent views on the origin of the planets, satellites, comets and other components of the solar system have been published. The author of this report first considered analysing these views here in brief form, but was forced to abandon this plan when it became clear that such an analysis, to be written convincingly, would require too much space. Since then he has published an analysis elsewhere (I ro).

Gerard P. Kuiper

President of the Commission

\section{REFERENCES}

(I) H. Camichel, Ann. Astrophys. 16, 4I, I953.

(2) A. Dollfus, C.R. Acad. Sci., Paris, 238, I475, 1954.

(3) A. Dollfus, C.R. Acad. Sci., Paris, 235, 1477, 1952.

(4) A. Dollfus, L'Astronomie, 68, 337, 1954.

(5) G. P. Kuiper, in The Atmospheres of the Earth and Planets, ed. G. P. Kuiper (2nd ed. Chicago 1952), pp. 307-8; and unpublished.

(6) G. P. Kuiper, Publ. A.S.P. 62, 133, 1950.

(7) D. Alter et al., Publ. A.S.P. 63, I74, $195 \mathrm{I}$.

(8) G. P. Kuiper, in The Atmospheres, ed. Kuiper, p. 387.

(9) I. Groeneveld and G. P. Kuiper, Ap. J. 120, 545-6, 1954.

(го) A. A. Nefedjev, Bull. Engelhardt Obs. no. 30, 3, 1953.

(I I) H. Camichel, Thèse, Paris, I954.

(12) G. P. Kuiper, in The Atmospheres, ed. Kuiper, p. 422.

(13) J. Ashbrook, Astr. J. 58, 145, 1953.

(14) G. P. Kuiper, $A p . J$. 120, 603, 1954.

(I5) A. Dollfus, $L ' A$ stronomie, 67, 66, 1953.

(16) Sir Harold Spencer Jones, in The Earth as a Planet (Chicago, 1954), chap. I.

(17) R. v. d. R. Woolley, Monthly Notices, 113, 521, 1953. 
(18) H. L. Giclas, Astr. J. 59, 128, 1954.

(19) I. Groeneveld and G. P. Kuiper, $A p . J .120,545-6,1954$.

(20) D. H. Menzel and F. L. Whipple, Astr. J. 59, 329, 1954.

(21) G. P. Kuiper, in The Atmospheres, ed. Kuiper, p. 371.

(22) V. V. Sharonov, Astr. Circ. U.S.S.R. no. 138, 7, 1953; Astr. J. U.S.S.R. 30, 532, 1953.

(23) V. V. Sharonov, Progress in Astronomy, 6, 181-249, 1954.

(24) N. P. Barabashev, Publ. Kharkov Obs. 2, 5, 1952.

(25) I. A. Parshin, Astr. Circ. U.S.S.R. no. 145, 12, 1954; Bull. Len. Univ. 9, no. 5, 85, 1954.

(26) N. P. Barabashev and A. T. Tchekirda, Publ. Kharkov Obs. 2, 9, 1952.

(27) A. Dollfus, C.R. Acad. Sci., Paris, 235, ror3, 1952.

(28) W. A. Baum and A. D. Code, Astr. J. 58, ro8, 1953.

(29) E. Pettit and R. S. Richardson, Publ. A.S.P. 65, 91, 1953.

(30) G. Reaves and M. F. Walker, Publ. A.S.P. 64, 15, 1952.

(3I) J. Dubois, L'Astronomie, 67, 44, 1953.

(32) J. Bouska and B. Ruzickova, Bull. Inst. astr. Czechosl. 4, 14, 1952.

(33) V. P. Dzhapiashvili, Bull. Abastumani Obs. no. 17, 29-47, 1954.

(34) A. V. Markov and D. E. Schegolev, Pulkovo Bull. 19, no. I5I, p. 34, 1953.

(35) V. M. Tchernov, Bull. Astr.-Geod. Soc. U.S.S.R. no. 14, 34-49, 1953.

(36) J. O'Keefe and J. P. Anderson, Astr. J. 57, ro8, 1952.

(37) S. L. Hess, $A p . J$. 118, 151, 1953.

(38) G. P. Kuiper, in The Atmospheres, ed. Kuiper, pp. 372-3, 381.

(39) A. Dollfus, C.R. Acad. Sci., Paris, 239, 954, 1954.

(40) G. P. Kuiper, in The Atmospheres, ed. Kuiper, pp. $38 \mathrm{gf}$.

(4I) J. Grandjean and R. M. Goody, $A p . J$. 121, 548, 1955.

(42) G. P. Kuiper, in The Atmospheres, ed. Kuiper, p. 36o.

(43) L. Goldberg, in The Earth as a Planet, ch. 9.

(44) B. Lyot, L'Astronomie, 67, 3-2 I, 1953.

(45) A. Dollfus, L'A stronomie, 67, 61, 85, 1953.

(46) A. Dollfus, $L^{\prime}$ A stronomie, 67, 63, 65, 1953.

(47) G. Fournier, L'A stronomie, 66, 355, 393, 490, 1952.

(48) G. A. Tikhov, 'L'hypothèse sur la paléobotanique de Mars et de Vénus', Bull. Acad. Kasakstan, I953.

(49) V. V. Sharonov, Bull. Len. Univ. 9, no. I I, 1954.

(50) N. P. Barabashev and A. T. Tchekirda, Astr. Circ. Kharkov Obs. no. II, 1953.

(5I) N. P. Barabashev, Astr. J. U.S.S.R. 29, 538, 1952; Astr. Circ. Kharkov Obs. no. 9.

(52) M. S. Bobrov, Astr. J. U.S.S.R. 29, 334, 1952; 31, 4I, 1954.

(53) M. V. Bannova, Ann. Len. Univ. no. I 53, I55, 1952.

(54) R. B. Leighton, Publ. A.S.P. 65, 197, 1953.

(55) E. P. Martz, Publ. A.S.P. 66, 45, 1954.

(56) A. Dollfus, L'Astronomie, 67, 72-5, 1953.

(57) 'D. B. McLaughlin, Astr. J. 59, 328, 1954; Publ. A.S.P. 66, 161, 22 I, 1954.

(58) G. P. Kuiper, Publ. A.S.P. 67, 280, 1955.

(59) E. Schoenberg, S.B. bayer, Akad. Wiss. I948.

(6o) E. Schoenberg and W. D. Heintz, Astr. Nachrichten, 282, 85, 1955.

(6I) G. P. Kuiper, The Atmospheres, p. $3^{84}$.

(62) G. P. Kuiper, $A p . J$. 120, 603, 1954.

(63) A. Dollfus, C.R. Acad. Sci., Paris, 234, 2046, 1952.

(64) M. J. Couture, L'Astronomie, 67, 5I, I953.

(65) G. P. Kuiper, Proc. Nat. Acad. Sci., Wash., 40, Io96, I954.

(66) K. Buettner, Publ. A.S.P. 64, I1, 1952.

(67) A. J. Wesselink, The Observatory, 74, 215, 1954.

(68) V. A. Fedoretz, Publ. Kharkov Obs. 2, 47-172, 1952.

(69) N. N. Sytinskaya, Astr. J. U.S.S.R. 30, 295-30I, 1953.

(70) N. P. Barabashev and A. T. Tchekirda, Publ. Kharkov Obs. 3, 13-25, 1954; Circ. Astr. Obs. Kharkov Univ. no. 12, 3-6, 1953. 
(71) A. V. Markov, Pulkovo Bull. 19, no. 149, 64-81, 1952.

(72) N. N. Sytinskaya, Astr. Circ. U.S.S.R. no. I44, I I-I2, 1953.

(73) V. V. Sharonov, Bull. Len. Univ. no. 30, 3-7, 1952.

(74) N. S. Orlova, Ann. Len. Univ. 153, I66-94, 1952; Bull. Len. Univ. no. 32, 3-4, I95 I.

(75) A. P. Borissova and N. A. Budnikova, Bull. Len. Univ. 8.

(76) V. V. Sharonov, Astr. J. U.S.S.R. 31, no. 5, 444-53, I954.

(77) A. V. Markov, Pulkovo Bull. r9, part 2, no. 149, p. 65, I952.

(78) I. Groeneveld and G. P. Kuiper, $A p . J$. 120, 200, 1954; $A p . J .120,529,1954$.

(79) A. V. Shatzel, $A p . J$. 120, 547, 1954.

(80) I. I. Ahmad, $A p$. J. 120, 55I, 1954.

(81) Ap.J. 120, 200, 1954.

(82) B. J. Levin, Astr. Circ. U.S.S.R. no. I4I, 1953.

(83) G. P. Kuiper, Proc. Nat. Acad. Sci., Wash., 39, II 59, 1953; Ap. J. 120, 2 19, 1954.

(84) O. J. Schmidt, C.R. Acad. Sci. U.R.S.S. 96, no. 3, 1954.

(85) E. A. Lubimova and A. S. Starkova, Astr. J. U.S.S.R. 31, no. 5, I954.

(86) G. P. Kuiper, Proc. Nat. Acad. Sci., Wash., 40, I Ior, I954.

(87) N. P. Barabashev, Investigation of the Physical Conditions on the Moon and the Planets (Kharkov Univ. 1952, 270 pp.).

(88) S. D. Gutshabash, Ann. Len. Univ. I, I53, 19-38, 1952.

(89) V. V. Sobolev, Progress in Astronomy, 6, 250-80, I954.

(9o) V. V. Sharonov, C.R. Acad. Sci. U.S.S.R. 82, no. 3, 55I-3; Astr. J. U.S.S.R. 29, $728-37,1952$.

(9I) V. V. Sharonov, Astr. Circ. U.S.S.R. no. 125, 8-9, 1952; Bull. Len. Univ. 8, no. 8, $5 \mathrm{I}-8 \mathrm{o}, 1953$.

(92) E. Rabe, Astr. J. 55, I I 2, 1950.

(93) H. C. Urey, Geochimica et Cosmochimica Acta, r, 23I, I95I ; G. P. Kuiper, The Atmospheres, p. 339.

(94) Harold Jeffreys, The Earth (3rd ed. Cambridge, I952).

(95) K. E. Bullen, Seismology (Methuen, 1954).

(96) Sir Edward Bullard and B. Mason, The Earth as a Planet, chs. 3 and 6.

(97) G. P. Kuiper, Proc. Nat. Acad. Sci., Wash., 40, 1096, 1954.

(98) W. H. Ramsey, Monthly Notices, Irr, 427, I95I.

(99) B. Miles and W. H. Ramsey, Monthly Notices, I12, 234, 1952.

(10o) W. C. De Marcus, Astr. J. 58, 36, 1953.

(Ior) M. J. M. Bernal and H. S. W. Massey, Monthly Notices, II4, I72, 1954.

(I02) H. P. Berlage, Proc. Kon. Ned. Acad. Wet. 54, 344, I95I.

(I03) H. C. Urey, The Planets, New Haven, I952, p. I59f.

(I04) G. P. Kuiper, in The Atmospheres, ed. Kuiper, p. 340.

(ro5) W. C. De Marcus, Astr. J. 59, I I6, I954.

(106) S. V. Kozlovskaya, C.R. Acad. Sci. U.R.S.S. 92, no. 5, 1953.

(107) E. A. Lubimova, Bull. Acad. Sci. U.R.S.S., Geophys. Ser., no. 2, 1952; no. 6, 1953.

(108) E. A. Lubimova, Publ. Geophys. Inst. 26, I954.

(Io9) J. A. Jacobs, Trans. R. Soc. Canada, 47, 33, I953; Ibid. 48, 33, I954; Nature, I 7 I, 835, 1953; Trans. Am. Geophys. Union, 35, I61, I954.

(1 10) G. P. Kuiper, J.R.A.S. Canada, 50, 57, 105, 158, 1956.

\section{APPENDIX}

\section{REPORT ON MARTIAN NOMENCLATURE}

At the Rome meetings Commission 16 formed a subcommittee, with $M$. Fournier as chairman, charged with formulating a simplified and adequate system for naming the Martian surface markings, both semi-permanent and transient (Trans. I.A.U. 8, 2I6-I7). M. Fournier prepared a ten-page memorandum which he sent on about I October r954 